Review paper

\title{
Imaging dose from cone beam computed tomography in radiation therapy
}

\author{
Parham Alaei ${ }^{\text {a, }}$, Emiliano Spezi ${ }^{\text {b, c }}$ \\ a University of Minnesota, Minneapolis, MN, USA \\ ${ }^{\mathrm{b}}$ School of Engineering, Cardiff University, Cardiff, Wales, UK \\ ${ }^{c}$ Velindre Cancer Centre, Cardiff, Wales, UK
}

\section{A R T I C L E I N F O}

\section{Article history:}

Received 17 February 2015

Received in revised form 29 May 2015

Accepted 5 June 2015

Available online 4 July 2015

\section{Keywords:}

Cone beam $\mathrm{CT}$

Imaging dose

kV CBCT

MV CBCT

\begin{abstract}
A B S T R A C T
Imaging dose in radiation therapy has traditionally been ignored due to its low magnitude and frequency in comparison to therapeutic dose used to treat patients. The advent of modern, volumetric, imaging modalities, often as an integral part of linear accelerators, has facilitated the implementation of imageguided radiation therapy (IGRT), which is often accomplished by daily imaging of patients. Daily imaging results in additional dose delivered to patient that warrants new attention be given to imaging dose. This review summarizes the imaging dose delivered to patients as the result of cone beam computed tomography (CBCT) imaging performed in radiation therapy using current methods and equipment. This review also summarizes methods to calculate the imaging dose, including the use of Monte Carlo (MC) and treatment planning systems (TPS). Peripheral dose from CBCT imaging, dose reduction methods, the use of effective dose in describing imaging dose, and the measurement of CT dose index (CTDI) in CBCT systems are also reviewed.
\end{abstract}

() 2015 Associazione Italiana di Fisica Medica. Published by Elsevier Ltd. This is an open access article under the CC BY-NC-ND license (http://creativecommons.org/licenses/by-nc-nd/4.0/).

\section{Introduction}

Cone beam computed tomography (CBCT) was developed in late 1990 s and put into clinical use in radiation therapy in the early 2000s. A CBCT system utilizes either the Megavoltage radiation beam delivered from the linear accelerator (linac), or a kilovoltage beam delivered using an additional x-ray tube mounted on the linac, to image the object from multiple projection angles and reconstruct a 3D image using the projected images. The benefit of CBCT, as opposed to the traditional 2D imaging using a portal imaging device, is volumetric visualization of the patient's body as well as enhanced visualization of soft tissue.

Due to the superiority of CBCT imaging in patient positioning, there has been a rapid growth of the use of these systems in radiation therapy, which has helped to make image-guided radiation therapy a routine technique employed worldwide. Currently, a variation of CBCT imaging is employed in Varian (Varian Medical Systems, Palo Alto, USA), Elekta (Elekta AB Stockholm, Sweden), Siemens (Siemens AG, Erlangen, Germany), and more recently, Vero (Brainlab AG, Feldkirchen, Germany) radiation delivery units.

\footnotetext{
* Corresponding author.

E-mail address: alaei001@umn.edu (P. Alaei).
}

Professional societies have recognized the growing use of this technology in routine clinical care and published guidelines for implementation and use of these systems. Among these are the "ACR-ASTRO Practice Guidelines for Image-Guided Radiation Therapy (IGRT)" [1], the "National Radiotherapy Implementation Group Report on Image Guided Radiotherapy (IGRT)" [2]published by the United Kingdom's National Cancer Programme Initiative, and the ASTRO white paper "Safety considerations for IGRT" [3]. Furthermore, there is recent indication that the use of IGRT may improve the clinical outcome of patients undergoing radiation therapy, which reinforces the use of image guidance [4].

The imaging dose delivered to patients from CBCT became an issue of concern soon after the start of clinical utilization of these systems. Even though imaging has been used for patient positioning since the onset of radiation therapy, the increased (often daily) frequency of imaging in the era of IGRT, as opposed to the traditional weekly portal imaging, and the potential inclusion of larger volumes of normal structures in $\mathrm{CBCT}$, raises the quantity of the dose delivered to the patient from imaging, as well as the integral dose. There is also the additional concern of increasing the chance of secondary cancers because of imaging dose. These stochastic risks have been recognized and estimated by the American Association of Physicists in Medicine (AAPM) Task Group 75 [5] and others [6,7]. Realizing the potential for significant imaging dose, 
many have investigated the magnitude of this dose by measurements and calculations. The distribution of papers published on imaging dose from CBCT per year is shown in Fig. 1. This figure only includes papers in peer-reviewed publications with complete methods and results sections.

In addition to the absorbed dose from CBCT, the use of different quality beams other than the therapeutic megavoltage ones raises questions as to the magnitude of effective dose delivered to patient, and the utilization of kilovoltage beams results in increased dose to the bones relative to soft tissue, which may be of concern in imaging of pediatric patients.

The availability and growing use of CBCT in radiation therapy has resulted in a large volume of publications in this field. In a previous publication Sykes [8] et al. reported on typical doses from commercial CBCT systems as well as on clinical consequences (risks and benefits) of using CBCT based IGRT. Although that work compiled useful data, it was not designed as a literature review. The aim of this systematic review was to find, categorize and summarize all high quality research data on measurements and calculation of the dose from CBCT imaging for both kilovoltage and Megavoltage imaging beams incorporated into radiotherapy linear accelerators.

There have been over 500 publications since early 2000s that include terms such as "cone beam CT" or "CBCT" and "radiation therapy" or "radiotherapy" as a key word, based on the advanced search performed using the engine Scopus.com (Elsevier B.V., The Netherlands). This review was conducted by searching the literature for all the work published on measurement and calculation of CBCT dose, including collection of beam data, modeling, estimation of peripheral and effective dose, and strategies to reduce dose. Of the 500 publications, only those that were relevant for this study, and included complete data and clear methods and results sections were included in this review.

Measured and calculated dose values are systematically reported for various systems and acquisition protocols. The variation of imaging dose with tissue density and body size, peripheral dose, dose reduction methods, and effective dose are also included in this work. The review also includes published data on measurements of CT Dose Index (CTDI) for CBCT systems with all the published values of CTDI methodically tabulated. The CTDI, although not a direct measure of patient dose, is a very commonly used index and its inclusion in this review was deemed beneficial for the professionals involved in the assessment of CBCT image quality and dose delivered using different systems and techniques.

This review could serve as a comprehensive source of data pertaining to various devices and techniques used in radiation therapy, helping clinicians and physicists with their decisions in routine clinical practice. The current review does not include information on the dose from fan-beam CT scanners, whether kilovoltage or Megavoltage (i.e. TomoTherapy (Accuray, Madison, USA)). It also does not include the topic of dose delivered to patients as a result of CBCT in diagnostic radiology, cardiology, and dentistry. Recent papers by Kalendar [9] and Dougeni et al. [10] provide a review of CT imaging doses in diagnostic radiology and other publications include dose values for Tomotherapy [11,12], C-arm CBCT [13], dental CBCT [14,15], and 4D CT systems [16].

This review is organized as follows: The first two sections focus on $\mathrm{CBCT}$ dose measurement and calculation, covering CBCT imaging dose measurements in phantom and patient, and CBCT imaging dose calculations, including beam data collection and modeling; Monte Carlo methods; use of treatment planning systems and other methods. The following sections review peripheral dose from CBCT imaging; CBCT imaging dose reduction, with special emphasis on pediatric cases; and the use of effective dose in $\mathrm{CBCT}$ imaging. The last section covers published data on CTDI measurements for CBCT systems. Each section consists of relevant data along with a discussion of the findings when appropriate.

\section{CBCT imaging dose measurements}

Among the papers reporting on dose measurements (excluding CTDI), $80 \%$ present results for phantom measurements and $20 \%$ present patient measurement data. Various detectors such as ion chamber, thermoluminiscent dosimeter (TLD), metal-oxide-semiconductor field-effect transistor (MOSFET), radiographic and radiochromic film, optically stimulated luminescence dosimeter (OSLD), and glass dosimeter have been used for this purpose, many of which produce challenges as to the detector calibration and response to low energy radiation when used for $\mathrm{kV}$ CBCT dosimetry. This warrants additional investigation into the suitability of the detector for these types of measurements, an example of which is the work done for OSLDs [17]. CBCT dose measurements were divided into two categories: phantom and patient studies and are summarized below.

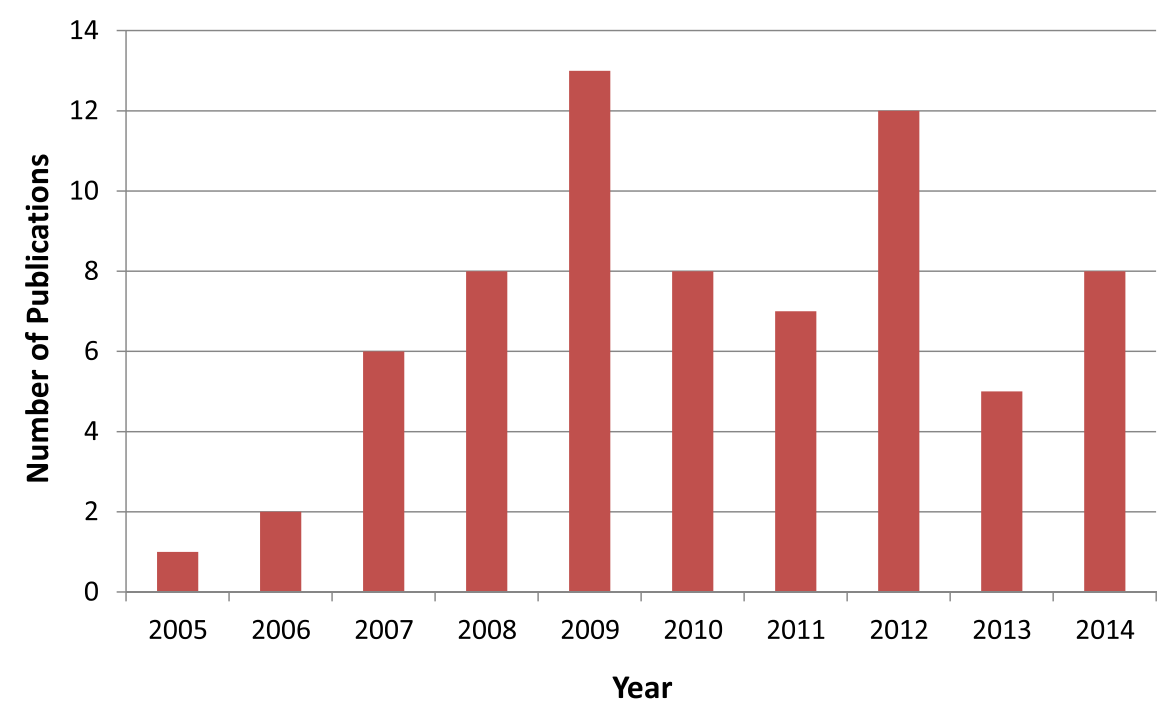

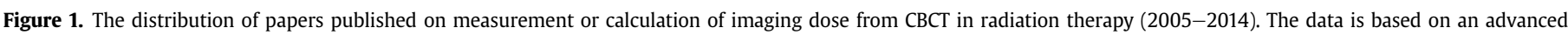
search of the Scopus.com bibliographic database. 


\section{Phantom studies}

The majority of work done on measuring the dose from CBCT imaging has been in phantoms for obvious reason of convenience and availability of them [6,18-44]. Of these, a large number have used anthropomorphic phantoms and TLDs as dosimeters, and others employed other types of phantoms and various detectors such as ion chamber, film, MOSFET, and OSLD. A summary of these can be found in Table 1. The doses measured range from 0.01 to 13 cGy per acquisition due to variations of imaging devices, type and size of phantom, location of measurement within phantom, and imaging techniques used. Due to the wide variation of experimental setups, it is hard to assign one value to the dose magnitude based on the anatomy imaged. In general, for the kilovoltage imaging, the low dose values are from head and neck imaging protocols that employ lower time-current products (mAs) and partial arc image acquisition whereas the higher ones are from trunk imaging with higher $\mathrm{mAs}$ values and full $360^{\circ}$ image acquisition. Megavoltage CBCT imaging using the $6 \mathrm{MV}$ beam (commonly called Therapy Beam Line or TBL) results in higher dose than kilovoltage one with a direct correlation to the imaging protocol (i.e. MU setting) used. However, the Megavoltage dose is often more evenly distributed within the body whereas the kilovoltage dose is more heterogeneously distributed and typically exhibits its maximum dose on the skin, and with the increased absorption in bone due to prominence of photoelectric effect.

\section{Patient studies}

The patient studies generally employed TLDs or other dosimeters to measure skin dose [20-22,27,45] although there have been two studies measuring the dose inside the rectum [46,47]. Patient dose measurements are summarized in Table 2. The skin dose measurements range from fraction of a cGy (for low dose head and neck imaging) to $7 \mathrm{cGy}$ (for high dose pelvic ones) and vary based on the measurement location, technique used, and patient size. These values are in the same range of those measured in phantom listed in Table 1 . The rectal dose measurements indicate 2-3 cGy average dose to rectum per CBCT acquisition for the protocol commonly used in clinical practice for pelvic imaging on the Elekta system.

\section{CBCT imaging dose calculations}

Imaging dose calculations often require beam data collection, modeling of the imaging systems, and verification of the accuracy of the model. In this section, we report on the studies that dealt with $\mathrm{CBCT}$ modeling and dose calculation.

\section{Beam data collection and modeling}

Imaging beam data for the kilovoltage units can be measured, simulated using Monte Carlo (MC) methods and, depending on the TPS used, modeled in the treatment planning system. Imaging beam data collection has its own challenges as compared to Megavoltage therapeutic ones as it is often difficult to position a water scanning system within the unit. Other challenges include low output of the imaging beams, which produce a low signal at depth and heating of the x-ray tube. For these reasons, beam data generation can be the result of a combination of measurements and MC simulations, with the latter being used to verify the accuracy of measurements.

MC simulation and verification of the imaging beams has been performed by Ding et al. $[48,49]$ for the Varian On Board Imaging (OBI) unit, and by Spezi [50] and Downes [51] for the Elekta X-ray Volume Imaging (XVI) unit, among others. Beam modeling in a treatment planning system for the OBI unit has been done by Alaei et al. [52] for the half-bowtie, half-fan acquisition, and by Alaei and Spezi [53] for most of the XVI imaging presets.

Megavoltage imaging employing the megavoltage treatment beam (Siemens) requires no beam data collection. As such, the imaging beam can easily be added to the treatment plans by adding an arc beam of $6 \mathrm{MV}$ photons. This has been done by several groups, indicating its feasibility and the ability of including MV CBCT dose in treatment planning $[54,55]$. The Siemens units, however, have the capability of cone beam imaging using two other modalities: 1) An "Imaging Beam Line" or IBL, which employs a degraded 4.2 MeV Megavoltage beam and a Carbon target to produce the imaging beam [56] and 2) The kVision kilovoltage imaging beam which employs a kilovoltage x-ray tube and image detector along the megavoltage beam line but in a reverse geometry. The IBL beam data has been collected and modeled in a treatment planning system by Flynn et al. [45] and similar work has been done by Dzierma et al. for the kVision system [57]. Table 3 contains a summary of beam data collection and simulation/modeling papers, noting that there are other publications in which some beam data collection is presented.

\section{Monte Carlo methods}

The MC method is regarded as a gold standard for modeling particle tracking and dose deposition in a wide range of energies of therapeutic and imaging interest. In the field of CBCT imaging dose calculations the EGSnrc code system, which includes the BEAMnrc and DOSXYZnrc codes, has been extensively used alongside other general purpose MC codes such as MCNP and Geant4 [48-51,58-71]. All publications found in our literature search reported 3D dose data calculated on voxelised geometries representing human anatomy, based on patient CT scans or virtual phantoms. Approximately half of the papers reported the use of phase space files generated at the exit window of the CBCT unit for each collimator setting used for imaging the patient. About $30 \%$ of the papers reported the use of a multiple source model of the CBCT unit and in the remainder particles were sampled from either a full simulation of the CBCT beam line (beam model) or using a simple photon spectrum. The Monte-Carlo studies have been summarized in Table 4. Due to high number of publications and the amount of data reported in these papers, it is not feasible to list all the organ dose data in this review. Hence we report in the dose summary column in Table 4 a cross-reference to the table number in the respective publication where the organ dose data can be found.

\section{Use of treatment planning system and other methods}

Computing and adding imaging dose to therapeutic one produces a complete picture of dose distribution in patient. This is easily achievable for imaging systems that utilize the therapeutic beam for imaging such as Megavoltage portal imaging and cone beam CT, even though this was never a routine practice in the era of port films and electronic portal imaging. Megavoltage cone beam CT adds considerable amount of dose to the treatment volume and surrounding tissue, hence its inclusion in the treatment plan was suggested early on and multiple papers report the dose to phantom and patient from MV CBCT imaging using different dose settings $[23,24,28,54,73-76]$. Similar work was performed for the imaging dose from Siemens IBL system, and compared to that of the original $6 \mathrm{MV}$ imaging beam (TBL) $[45,77]$.

Inclusion of kilovoltage imaging beam in the treatment plan, however, requires beam data collection and modeling. Some of the work done in this area is listed in Table 3, which only applies to Pinnacle treatment planning system (Philips Medical Systems, Milpitas, CA) used for imaging beam modeling and dose 
Table 1

Summary of CBCT dose measurements in phantom. The version of the imaging system is only included when available.

\begin{tabular}{|c|c|c|c|c|c|c|}
\hline $\begin{array}{l}\text { Kilovoltage } \\
\text { CBCT }\end{array}$ & $\begin{array}{l}\text { Manufacturer (Version, } \\
\text { if specified) }\end{array}$ & $\mathrm{kVp}$ & mAs/acquisition & Phantom type & Dosimeter & $\begin{array}{l}\text { Dose in phantom/ } \\
\text { fraction (cGy) }\end{array}$ \\
\hline Sykes [18] & Elekta XVI (v3.1) & 130 & 0.56 & Rando head & TLD & $1.9-2.9$ \\
\hline \multirow{3}{*}{ Islam [20] } & Elekta XVI & 100 & 2 & Cylindrical (16 \& 30 cm dia.) & Chamber & $0.7-3.5$ \\
\hline & & 120 & 2 & & & \\
\hline & & 140 & 2 & & & \\
\hline \multirow[t]{3}{*}{ Amer [21] } & Elekta XVI & 100 & 0.1 & Rando head & TLD & 0.13 \\
\hline & & 120 & 0.4 & Rando chest & & 0.72 \\
\hline & & 130 & 1.2 & Rando pelvis & & 2.1 \\
\hline Wen [22] & Varian OBI & 125 & 2 & Rando pelvis & TLD & $2.1-4.7$ \\
\hline \multirow[t]{2}{*}{ Kan [6] } & Varian OBI & 125 & 2 & Female Rando head, chest, pelvis & TLD & $3.6-6.7$ \\
\hline & & 125 & 0.4 & & & \\
\hline Osei [25] & Varian OBI & 125 & 2 & Rando pelvis & TLD & $3.0-11.5$ \\
\hline Winey $[26]$ & Varian OBI & 125 & 1.6 & CIRS Thorax & Chamber/OSL & $2.4-9.1$ \\
\hline Marinello [27] & Varian OBI & 125 & 2 & Rando & TLD/Gafchromic & $4.7-6.2$ \\
\hline \multirow[t]{5}{*}{ Tomic [29] } & Varian OBI (v1.4) & 100 & 0.2 & Rando head, chest, pelvis & Gafchromic & $0.1-4.7$ (surface) \\
\hline & & 100 & 0.4 & & & \\
\hline & & 100 & 1.6 & & & \\
\hline & & 125 & 1.04 & & & \\
\hline & & 125 & 1.6 & & & \\
\hline \multirow[t]{5}{*}{ Tomic [30] } & Varian OBI (v1.4) & 100 & 0.2 & Rando head, chest, pelvis & Gafchromic & $0.03-2.8$ (surface) \\
\hline & & 100 & 0.4 & & & \\
\hline & & 100 & 1.6 & & & \\
\hline & & 125 & 1.04 & & & \\
\hline & & 125 & 1.6 & & & \\
\hline \multirow[t]{6}{*}{ Hyer [31] } & Varian OBI (v1.4) & 100 & 0.4 & Male Anthro head, chest, pelvis & Scintillator & $0.2-2.8$ \\
\hline & & 110 & 0.4 & & & \\
\hline & & 125 & 1.04 & & & \\
\hline & Elekta XVI (v.4) & 100 & 0.1 & & & $0.1-2.9$ \\
\hline & & 120 & 1.6 & & & \\
\hline & & 120 & 2.56 & & & \\
\hline \multirow[t]{7}{*}{ Palm [32] } & Varian OBI (v1.3) & 125 & 0.4 & Female Rando head, chest, pelvis & TLD & $8.7-13$ \\
\hline & & 125 & 2 & & & \\
\hline & Varian OBI (v1.4) & 125 & 1.04 & & & $0.25-3.42$ \\
\hline & & 125 & 2 & & & \\
\hline & & 100 & 0.2 & & & \\
\hline & & 100 & 0.4 & & & \\
\hline & & 100 & 2 & & & \\
\hline \multirow[t]{3}{*}{ Cheng [33] } & Varian OBI & 125 & 1.6 & Female Rando head \& pelvis & TLD & $1.3-9.4$ \\
\hline & & 100 & 1.6 & & & $0.4-3.0$ \\
\hline & & 124 & 1.04 & & & \\
\hline Dufek [34] & Varian OBI (v1.3) & 125 & 264 (total) & Rando head \& pelvis & TLD & $0.01-1.66$ \\
\hline & Varian OBI (v1.4) & 125 & 0.64 & & & $0.01-1.19$ \\
\hline & & 100 & 0.2 & & & \\
\hline & Elekta XVI & 100 & 0.1 & & & $0.01-3.49$ \\
\hline & & 120 & 1.6 & & & \\
\hline Halg [39] & Varian OBI (v1.4) & 125 & 2.0 & Rando & TLD & $0.7-2.8$ \\
\hline & & 125 & 2.0 & & & $0.7-4.0$ \\
\hline & & 110 & 0.4 & & & $0.2-0.8$ \\
\hline & & 100 & 2.0 & & & $0.3-1.8$ \\
\hline & Varian TrueBeam (v1.0) & 125 & 686.4 (total) & & & $0.4-1.7$ \\
\hline & Elekta XVI (v4.2) & 120 & 2.6 & & & $0.7-3.9$ \\
\hline Giaddui [41] & Elekta XVI (v4.5) & 100 & 0.4 & Rando head, chest, pelvis & Gafchromic/OSL & $0.02-5$ \\
\hline & & 100 & 0.4 & & & \\
\hline & & 120 & 0.32 & & & \\
\hline & & 120 & 1.6 & & & \\
\hline & Varian OBI (v1.5) & 100 & 0.72 & & & $0.4-5.6$ \\
\hline & & 125 & 0.4 & & & \\
\hline & & 125 & 1.6 & & & \\
\hline & & 125 & 3.6 & & & \\
\hline Alvarado [42] & Varian OBI (v1.5) & 110 & 0.4 & Female Rando chest & Gafchromic & $0.8-1.0$ \\
\hline Moon [43] & Elekta XVI & 120 & 1.6 & Female Rando head, chest, pelvis & Glass & $0.2-3.0$ \\
\hline Nobah [44] & Varian OBI (v1.4) & 100 & 0.4 & Rando head, chest, pelvis & Gafchromic & $0.03-4.91$ \\
\hline & & 110 & 0.4 & & & \\
\hline & & 125 & 1.6 & & & \\
\hline & Varian TrueBeam & 100 & 0.4 & & & $0.07-3.15$ \\
\hline & & 125 & 0.4 & & & \\
\hline & & 125 & 1.6 & & & \\
\hline Megavoltage СВСТ & & MU & & & & \\
\hline Gayou [23] & Siemens TBL & $5,8,10,15$ & & Rando head \& pelvis & TLD/Radiographic film & $6.0-7.3$ in $\mathrm{HN}(8 \mathrm{MU})$ \\
\hline & & & & & & $9.9-12.1$ in Pelvis (15 MU) \\
\hline & & 10 & & Cylindrical (16 and 32 dia.) & Chamber & $7.9 \& 9.2$ in center \\
\hline Morin [24] & Siemens TBL & 5,9 & & Cylindrical & Chamber/MOSFET & Not reported \\
\hline Isambert [28] & Siemens TBL & 5 & & Cylindrical & Chamber & 3.7 \\
\hline & & & & Rando & TLD & 3.7 (isocenter) \\
\hline Quinn [36] & Siemens TBL & 8 & & Female Anthro & TLD/MOSFET & $0.1-4.5$ on skin \\
\hline Halg [39] & Siemens TBL & 3 & & Rando & TLD & $0.9-2.4$ \\
\hline
\end{tabular}


Table 2

Summary of CBCT dose measurements in/on patient.

\begin{tabular}{|c|c|c|c|c|c|}
\hline Kilovoltage CBCT & Manufacturer & $\mathrm{kVp}$ & mAs/acquisition & Dosimeter & Dose/fraction (cGy) \& location \\
\hline Islam [20] & Elekta XVI & 120 & 2 & MOSFET & $1.12-1.84$ (skin) \\
\hline \multirow[t]{3}{*}{ Amer [21] } & Elekta XVI & 100 & 0.1 & TLD & 0.12 (skin) \\
\hline & & 120 & 0.4 & & $0.6-1.1$ (skin) \\
\hline & & 130 & 1.2 & & $2.2-3.5$ (skin) \\
\hline Walter [46] & Elekta XVI & 120 & 1 & Chamber & 1.72 (avg.) (rectum) \\
\hline Wen [22] & Varian OBI & 125 & 2 & TLD & $2.3-6.1$ (skin) \\
\hline Jeng [47] & Elekta XVI & 120 & 1 & TLD & 2.86 (avg.) (rectum) \\
\hline \multirow{2}{*}{ Marinello [27] } & Varian OBI & 125 & 2 & TLD & $5.8-7.3($ skin, $\mathrm{AP} / \mathrm{PA})$ \\
\hline & & & & & $3.4-4.5$ (skin, lateral) \\
\hline
\end{tabular}

Table 3

Summary of CBCT beam data collection and simulation/modeling.

\begin{tabular}{|c|c|c|c|}
\hline Kilovoltage CBCT & System & Beams & Details \\
\hline Ding [49] & Varian OBI & Single beam, half-fan, half-bowtie & Beam data collection, MC simulation and verification \\
\hline Chow [58] & Elekta XVI & Single cassette, no filter & Beam data collection, MC simulation and verification \\
\hline Spezi [50] & Elekta XVI & Multiple cassettes, no filter & Beam data collection, MC simulation and verification \\
\hline Downes [51] & Elekta XVI & Multiple cassettes, bowtie & Beam data collection, MC simulation and verification \\
\hline Ding $[48]$ & Varian OBI & Multiple beams/filters & Beam data collection, MC simulation and verification \\
\hline Alaei [52] & Varian OBI & Single beam, half-fan, half- bowtie & Modeling in TPS and verification \\
\hline Deng [59] & Varian OBI & Half-fan, half-bowtie \& Full-fan, full-bowtie & Beam data collection, MC simulation and verification \\
\hline Deng [60] & Varian OBI & Half-fan, half-bowtie \& Full-fan, full-bowtie & Beam data collection, MC simulation and verification \\
\hline Alaei [53] & Elekta XVI & Multiple cassettes, no filter and bowtie & Modeling in TPS and verification \\
\hline Dzierma [57] & Siemens kView & Two beam qualities & Beam data collection, modeling in TPS and verification \\
\hline \multicolumn{4}{|l|}{ Megavoltage СВСТ } \\
\hline Flynn [45] & Siemens IBL & IBL beam & Beam data collection, modeling in TPS and verification \\
\hline
\end{tabular}

Table 4

Summary of CBCT Monte Carlo dose calculations.

\begin{tabular}{|c|c|c|c|c|c|c|c|}
\hline & Manufacturer & $\begin{array}{l}\text { Acquisition } \\
\text { techniques per frame }\end{array}$ & Code used & Dose type & Source type & Phantom type & Dose summary \\
\hline Chow [58] & Elekta XVI & $\begin{array}{l}100,120,130 \text {, } \\
140 \mathrm{kVp} \text {, unspecified } \\
\mathrm{mAs}\end{array}$ & EGSnrc/BEAMnrc & Medium & Phase space & Patient CT & Table IV (organ doses) \\
\hline Ding [49] & Varian OBI & $125 \mathrm{kVp}, 2 \mathrm{~mA} \mathrm{~s}$ & BEAMnrc/DOSXYZnrc & Medium & Phase Space & $\begin{array}{l}\text { RSVP phantom and } \\
\text { patient CT }\end{array}$ & Table I (organ doses) \\
\hline Gu [61] & $\mathrm{N} / \mathrm{A}$ & $\begin{array}{l}125 \mathrm{kVp}, 2 \mathrm{~mA} \mathrm{~s}- \\
6 \mathrm{MV} 0.03 \mathrm{MU}\end{array}$ & MCNPX & Medium & X-ray Spectrum & VIP Man phantom & $\begin{array}{l}\text { Table } 5(\mathrm{kV}) \text { Table } 8 \\
(\mathrm{MV})\end{array}$ \\
\hline Downes [51] & Elekta XVI & $120 \mathrm{kVp}, 1.6 \mathrm{~mA} \mathrm{~s}$ & EGSnrc/BEAMnrc & Water & Phase Space & $\begin{array}{l}\text { Phantoms and patient } \\
\text { CT }\end{array}$ & $\begin{array}{l}\text { Figures 10-12 (organ } \\
\text { doses) }\end{array}$ \\
\hline Walters [62] & Varian OBI & $125 \mathrm{kVp}, 2 \mathrm{mAs}$ & BEAMnrc & Medium & Phase Space & $\begin{array}{l}\text { Voxelized human } \\
\text { phantoms }\end{array}$ & $\begin{array}{l}\text { Table II (skeletal } \\
\text { doses) }\end{array}$ \\
\hline Ding [63] & Varian OBI & $125 \mathrm{kVp}, 2 \mathrm{mAs}$ & DOSXYZnrc & Medium & Phase Space & Patient CT & Table 1 (organ doses) \\
\hline Ding [64] & Varian OBI & $\begin{array}{l}125 \mathrm{kVp}, 250 \mathrm{~mA} \mathrm{~s} \\
\text { (total) }\end{array}$ & MCNPX & Medium & X-ray Spectrum & Patient CT & Table I (organ doses) \\
\hline Ding [48] & Varian OBI & $\begin{array}{l}100 \mathrm{kVp}, 0.2,0.4 \\
2 \mathrm{~mA} \mathrm{~s} ; 125 \mathrm{kVp}, 0.4, \\
1.04,2 \mathrm{~mA} \mathrm{~s}\end{array}$ & DOSXYZnrc & Medium & Phase Space & Patient CT & \\
\hline Qiu [65] & Varian OBI & $\begin{array}{l}125 \mathrm{kVp} \text {, unspecified } \\
\mathrm{mAs}\end{array}$ & BEAMnrc/DOSXYZnrc & Medium & Full head & Patient CT & $\begin{array}{l}\text { Tables } 1 \text { and } 2 \text { (organ } \\
\text { doses) }\end{array}$ \\
\hline Spezi [66] & Elekta XVI & $\begin{array}{l}100 \mathrm{kVp}, 0.1 \mathrm{~mA} \mathrm{~s} \\
120 \mathrm{kVp}, 1.6 \mathrm{~mA} \mathrm{~s}\end{array}$ & EGSnrc/BEAMnrc & Medium & Phase Space & Patient CT & Table 2 (organ doses) \\
\hline Deng [59] & Varian OBI & $\begin{array}{l}60,80,100,125 \mathrm{kVp} \\
1.04,1.6 \mathrm{~mA} \mathrm{~s}\end{array}$ & EGS4/BEAM/MCSIM & Medium & Source Model & Patient CT & Table 2 (organ doses) \\
\hline Deng [60] & Varian OBI & $\begin{array}{l}60,80,100,125 \mathrm{kVp} \\
1.04,1.6 \mathrm{~mA} \mathrm{~s}\end{array}$ & EGS4/BEAM/MCSIM & Medium & Source Model & Patient CT & $\begin{array}{l}\text { Table } 3 \text { (pediatric } \\
\text { organ doses) }\end{array}$ \\
\hline Qiu [67] & Varian OBI & $125 \mathrm{kVp}, 1.04 \mathrm{~mA} \mathrm{~s}$ & BEAMnrc/DOSXYZnrc & Medium & Full head & Patient CT & \\
\hline Zhang [68] & Varian OBI & $\begin{array}{l}100 \mathrm{kVp}, 2 \mathrm{~mA} \mathrm{~s} \\
125 \mathrm{kVp}, 1.04 \mathrm{~mA} \mathrm{~s}\end{array}$ & EGS4/BEAM/MCSIM & Medium & Source Model & Patient CT & \\
\hline Ding [69] & Varian OBI & $\begin{array}{l}100,110,125 \mathrm{kVp} \\
0.36-3.7 \mathrm{~mA} \mathrm{~s}\end{array}$ & EGS4/BEAM/MCSIM & Medium & Source Model & Patient CT & \\
\hline Son [70] & Varian OBI & $\begin{array}{l}125 \mathrm{kVp}, 2.0 \& \\
0.4 \mathrm{~mA} \mathrm{~s}\end{array}$ & BEAMnrc & Medium & Phase Space & Patient CT & \\
\hline Montanari [72] & Varian OBI & $\begin{array}{l}100 \mathrm{kVp} 0.4 \mathrm{~mA} \mathrm{~s} \\
125 \mathrm{kVp}, 1.04 \\
2.0 \mathrm{~mA} \mathrm{~s}\end{array}$ & gCTD & Medium & Source Model & $\begin{array}{l}\text { Phantoms and } \\
\text { patient } \mathrm{CT}\end{array}$ & $\begin{array}{l}\text { Tables 4-6 (organ } \\
\text { doses) }\end{array}$ \\
\hline
\end{tabular}




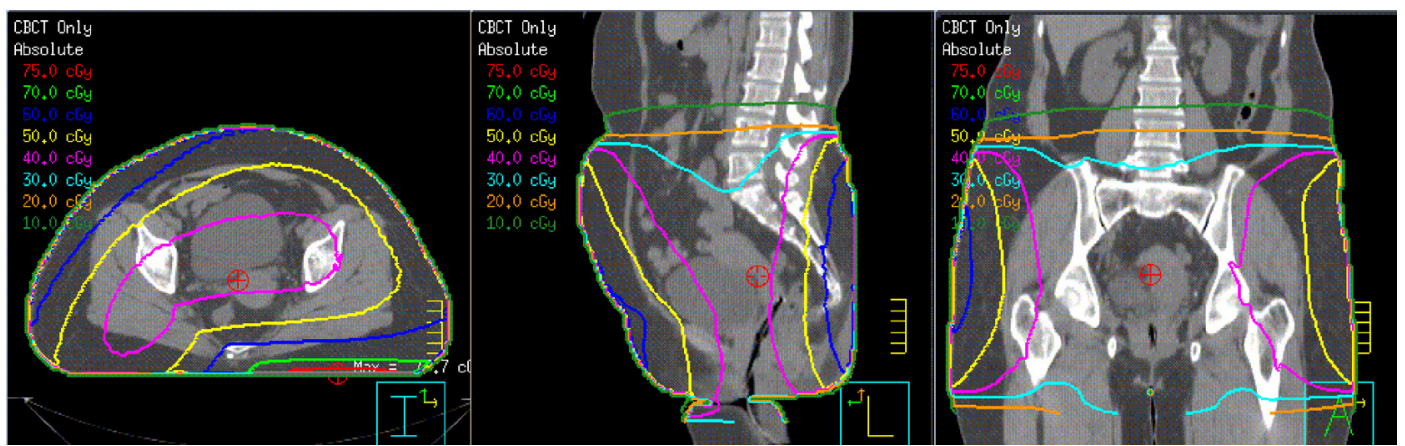

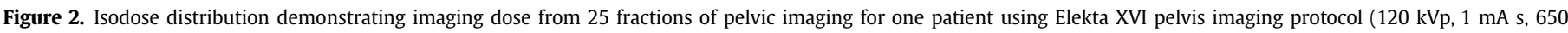
projections) and calculated using Pinnacle treatment planning system. (Reproduced with permission from Alaei et al. [78]).

computation [52,53,57,78]. Fig. 2 is an example of imaging dose distribution in pelvis calculated by the TPS. Due to algorithm limitations in this system and its applicability to Megavoltage beam energies, this computation produces dose distributions which are of reasonable accuracy in soft tissue and lung, but not in and around bone mineral. Adding the dose distribution generated by different quality beams is essentially summing the physical doses delivered from Megavoltage and kilovoltage beams. Since there is currently no accurate method to convert absorbed dose to effective dose for different quality beams, all the doses reported in these publications and this review are absorbed dose.

In addition to the use of treatment planning system and MC, other methods have also been proposed to compute imaging dose. One is a correction-based dose calculation algorithm [79-81]. This algorithm overcomes the inaccuracy of convolution-based dose calculation algorithms, due to not accounting for atomic number changes in kilovoltage energy range, by introducing another correction factor to account for these changes.
Another method extracts organ doses from CTDI values by introducing an organ dose conversion coefficient for clinical imaging protocols used [82]. In yet another dose estimation method, MC calculations have been used to calculate organ doses for different cohorts of patients imaged using various clinical protocols. This data has then been tabulated to be used to estimate organ doses for patients imaged using the same protocols [83]. The rationale for this dose estimation method is the relatively small variation of imaging dose with patient size and isocenter position; hence the approach taken is different than those attempting to calculate individualized imaging dose and including it in the treatment plan.

All the non-Monte Carlo dose calculation methods are tabulated in Table 5.

A few observations on CBCT dose calculations are warranted: MV CBCT exhibits a dose gradient within the body because of the partial arc image acquisition employed as seen in Fig. 3; hence for a patient treated in supine position, the bulk of the dose is deposited in the anterior part of the body.

Table 5

Summary of CBCT dose calculations performed by methods other than Monte Carlo.

\begin{tabular}{|c|c|c|c|c|c|c|}
\hline Kilovoltage CBCT & Manufacturer & $\mathrm{kVp}$ & mAs/acquisition & Phantom type & Method & Notes \\
\hline Alaei [52] & Varian OBI & 120 & 2 & Rando Pelvis & Pinnacle TPS ${ }^{d}$ & a \\
\hline Alaei [53] & Elekta XVI & 100,120 & $0.1,0.25,1,1.6,2.56$ & Rando head, chest, pelvis & Pinnacle TPS & $\mathrm{a}$ \\
\hline Alaei [78] & Elekta XVI & 100,120 & $0.1,1$ & Patient & Pinnacle TPS & b, c \\
\hline Dzierma [57] & Siemens kView & 70,121 & $0.5,0.6,1$ & Rando head, chest, pelvis & Pinnacle TPS & $\mathrm{a}, \mathrm{b}$ \\
\hline Ding [49] & Varian OBI & $\mathrm{N} / \mathrm{A}$ & $\mathrm{N} / \mathrm{A}$ & N/A & Correction-based algorithm & a \\
\hline \multirow[t]{2}{*}{ Hyer [82] } & Varian OBI & $100,110,125$ & $0.4,1.04$ & CTDI/Anthro & 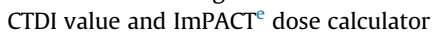 & a \\
\hline & Elekta XVI & 100,120 & $0.1,1.6,2.56$ & & & \\
\hline Pawlowski [80] & Varian OBI & $\mathrm{N} / \mathrm{A}$ & $\mathrm{N} / \mathrm{A}$ & $\mathrm{N} / \mathrm{A}$ & Correction-based algorithm & $\mathrm{a}, \mathrm{b}$ \\
\hline Megavoltage СВСТ & & MU & & & & \\
\hline Peng [73] & Siemens TBL & 9 & & Patient & Pinnacle TPS & $\mathrm{b}$ \\
\hline Gayou [23] & Siemens TBL & $5,8,10,15$ & & Cylindrical/Rando & Xio TPS & $\mathrm{a}$ \\
\hline Miften [54] & Siemens TBL & 15 & & Patient & Xio TPS & b, c \\
\hline Morin [55] & Siemens TBL & 5,9 & & Cylindrical/patient & Pinnacle TPS & a, b, c \\
\hline Isambert [28] & Siemens TBL & 5 & & $\begin{array}{l}\text { Cylindrical/Rando/ } \\
\text { patient }\end{array}$ & ISOgray TPS $^{\mathrm{h}}$ & a, b \\
\hline Flynn [45] & Siemens TBL \& IBL & & & Cylindrical/patient & Pinnacle TPS & $a, b$ \\
\hline Beltran [77] & Siemens TBL \& IBL & $\mathrm{N} / \mathrm{A}$ & & Solid water/patient & PlanUNC TPS ${ }^{g}$ & a, b \\
\hline vanAntwerp [74] & Siemens TBL & $2,4,8$ & & Patient & Pinnacle TPS & $\mathrm{b}$ \\
\hline Akino [75] & Siemens TBL & $3,5,8,15$ & & I'mRT phantom/patient & Xio TPS & a, b, c \\
\hline Zabel-du Bois [76] & Siemens TBL & 6,10 & & Patient & VOXELPLAN TPS ${ }^{\mathrm{i}}$ & $\mathrm{b}$ \\
\hline
\end{tabular}

\footnotetext{
a Accuracy verified in phantom.

b Patient dose calculation.

c Imaging dose combined with treatment planning dose.

d Philips Medical Systems, Eindhoven, The Netherlands.

e ImPACT Group, St. George's Healthcare NHS Trust, London, UK.

f Elekta AB, Stockholm, Sweden.

g University of North Carolina, NC, US.

h Dosisoft, Cachan, France.

i DKFZ, Heidelberg, Germany.
} 


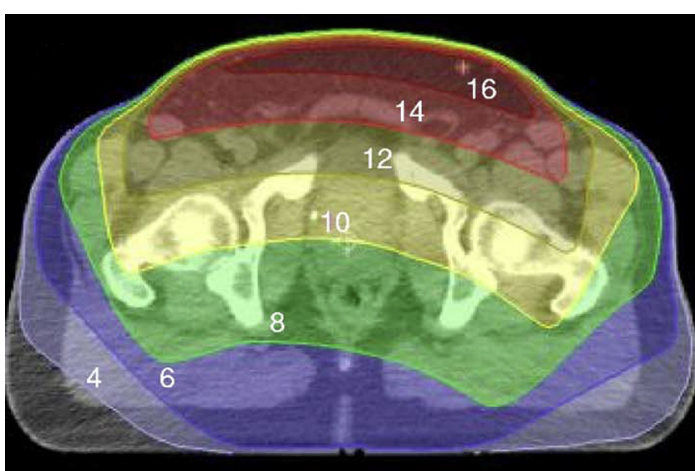

Figure 3. Distribution of dose deposited in the pelvis by a single fraction of MV CBCT imaging for a prostate patient, with $10 \mathrm{cGy}$ at isocenter. The isodose lines are labeled in cGy. (Reproduced with permission from Miften et al. [54]).

The kilovoltage imaging beams, on the other hand, deposit their maximum dose on the skin with enhanced dose absorption in bone mineral due to predominance of photoelectric effect. Fig 4 is an example of an MC-calculated $\mathrm{kV} \mathrm{CBCT}$ dose distribution.

Patient size does play a role in the imaging dose from $\mathrm{kV} \mathrm{CBCT}$ with larger patients receiving lower dose to the isocenter and internal organs, but not to the skin [78]. This can be described as a correlation between body mass index (BMI) and organ doses, especially in pelvic imaging. This correlation is weak in the case of Megavoltage imaging [74] but stronger for kilovoltage ones [78]. The correlation between patient size and imaging dose translates into increased dose to pediatric patients when adult imaging techniques are employed. This is further discussed in the section on dose reduction in pediatric cases below.

\section{Peripheral dose from CBCT imaging}

As in any other application of $\mathrm{x}$ rays, there is always dose deposition outside the volume of interest, with its magnitude depending on the amount of leakage and scatter. The peripheral dose from imaging could be of interest when there is a need to assess and/or minimize the dose outside the treatment volume, for example when the patient has a Cardiac Implantable Electronic Device (CIED).

Although many have pointed to out-of-field dose from СВCT imaging, few publications have exclusively focused on the peripheral dose. In one such article, Perks et al. [84] measured the peripheral dose from $\mathrm{kV} \mathrm{CBCT}$ and compared it to that from radiation

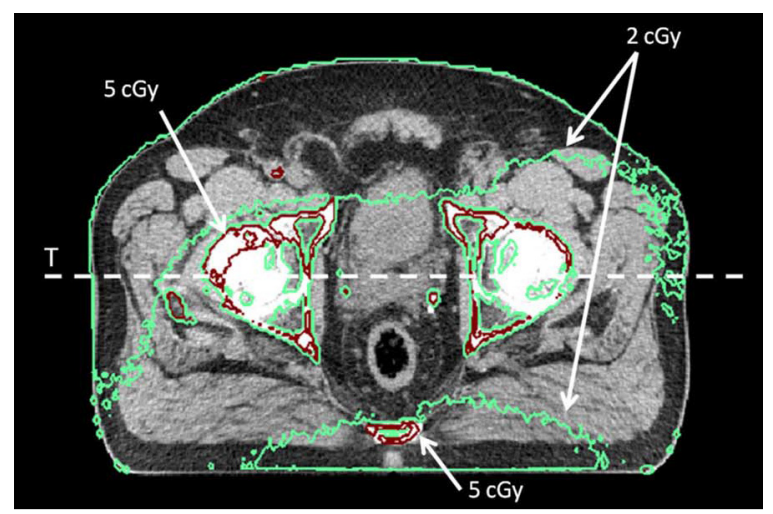

Figure 4. Patient dose from Elekta XVI CBCT pelvis scan simulated using the M10 collimator and F1 (bowtie) filter. (Reproduced with permission from Downes et al. [51]). therapy treatment itself by surface and internal phantom measurements. They concluded that peripheral doses from imaging, at measurement points of equal distance from the central axis, are of the same order of magnitude as those of an IMRT treatment. Qiu et al. [67] also investigated the magnitude of peripheral dose from $\mathrm{kV}$ CBCT as well as IMRT delivery and linac head leakage using MC simulation and concluded that dose to peripheral organs from CBCT is of the same order of magnitude as linac leakage and one order of magnitude lower than IMRT or RapidArc scatter dose.

In both of the above studies the imaged volume is larger than the treated one and the CBCT dose at a few centimeters from the edge of imaged volume drops to fractions of cGy. In addition, both studies utilized the pelvic (high dose) imaging hence the peripheral dose could be substantially less if a low dose setting is used. In another study, Jia et al. [85] measured the peripheral dose from a MV CBCT system and concluded that the magnitude of its peripheral dose is similar to that of $\mathrm{kV} \mathrm{CBCT}$ imaging, and that it increases with use of higher dose imaging.

\section{CBCT imaging dose reduction}

There are methods to reduce the imaging dose such as reducing the number of frames acquired and/or decreasing the current-time (mAs) settings for a particular acquisition in kilovoltage imaging. These reductions, however, may compromise the image quality. There are several studies examining this such as those by Sykes et al. [18], Ding et al. [86] and Alvarado et al. [42] in which the authors reduced the number of image acquisitions, adjusted the start/stop angles of the imaging source, reduced scan length, and used low-dose (or reduced mAs) protocols to image instead of standard high-dose ones. Another method to reduce imaging dose proposed by Roxby et al. [87] introduces a Copper filter to the beam hence increasing its half value layer and decreasing the delivered dose. More recently, Grelewicz and Dzierma [88] proposed a method to reduce $\mathrm{kV}$ imaging dose by combining $\mathrm{MV}$ and $\mathrm{kV}$ inverse planning using MC. As for the megavoltage beams, the use of low accelerating potential for the imaging beam and a Carbon target, the IBL, has reduced the dose commonly encountered when using the $6 \mathrm{MV}$ therapeutic beam for imaging [56]. Many of the above methods can easily be implemented in the clinic.

\section{Dose reduction in pediatric cases}

Imaging dose reduction is of greater importance in children than adults due to several factors. First is the increased susceptibility of children to radiation-induced secondary malignancies. The second factor is the increased absorption of kilovoltage beams in bone mineral, which could have a detrimental effect on the bone growth in children. And the third is that using standard CBCT imaging protocols often result in unnecessarily excessive dose to children due to their smaller body sizes.

In a Monte Carlo study, Ding and Coffey [63] showed that the pediatric imaging dose delivered to several organs is up to twice that of adult imaging. This ratio is higher for dose to the bone. Zhang et al. [68] evaluated $\mathrm{kV}$ CBCT doses to pediatric patients of various sizes and noted a three-fold variation of mean dose to femurs in their study population due to patient size differences. Therefore, imaging dose to patients of smaller size is enhanced should the same imaging protocols as adults are utilized. In another Monte Carlo study, Deng et al. [59] calculated kV CBCT imaging dose to pediatric cancer patients and concluded that critical structures in children receive doses larger by a factor of 2-3 compared to adult ones (Fig. 5). All of these studies verify the correlation between BMI and organ doses discussed previously. 

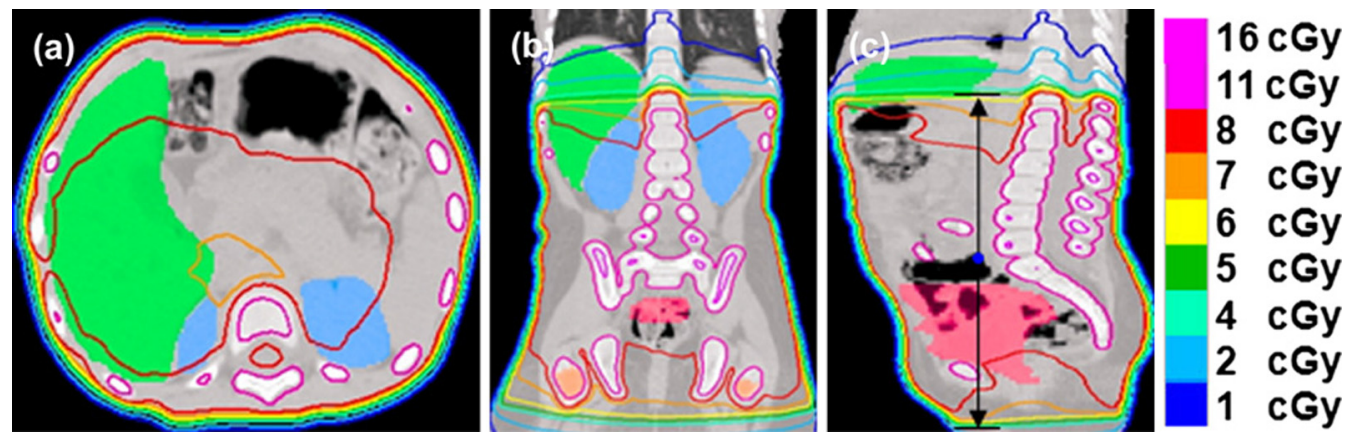

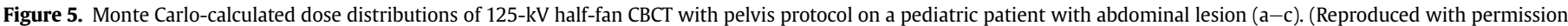
from Deng et al. [59]).

Considering the increased imaging dose delivered to pediatric patients, dose reduction methods should be employed to manage these cases. One method to achieve this is utilizing protocols used for adult head and neck imaging for body imaging of pediatric patients (essentially decreasing the current-time product), or reducing the number of frames [89]. Using these methods, the imaging dose to patient is shown to decrease by several fold as shown in Fig. 6. Other potential dose reduction methods include modifying start/stop angles of imaging beams both to reduce the dose and to avoid critical structures. All of these methods could be used providing the image quality is not compromised.

\section{Estimation of effective dose from CBCT imaging}

The AAPM Task Group 75 report [5] emphasized the use of effective dose when evaluating patient exposure to imaging doses. Several studies reported on the effective doses from kilovoltage СВCT $[6,31,33,34,39,43,90]$. In all these cases, the absorbed dose was measured in an anthropomorphic phantom and converted to effective dose using the tissue weighting factors obtained from
International Commission on Radiological Protection (ICRP) reports 60 and 103 [91,92]. They reported effective dose values within a wide range of 1.1-24 $\mathrm{mSv}$ for trunk and 0.04-9.4 $\mathrm{mSv}$ for head and neck imaging, per fraction. In another study, Gu et al. [61] computed effective doses for both $\mathrm{kV}$ and MV imaging using Monte Carlo-calculated absorbed doses and ICRP 103 weighting factors and reported values within the above range.

The wide variation of reported values can be explained based on the anatomical site, measured dose, relative tissue weighting factors used, imaging protocol (low vs. standard dose), and make of the imager which dictates the extent of the volume imaged, as well as use of bowtie filter. The version of the hardware/software utilized also affects the outcome, with newer versions tending to optimize image quality and size, which contributes to reduce the concomitant dose. In general, low dose imaging protocols employed in head and neck imaging result in effective doses less than $2 \mathrm{mSv}$. Effective doses of up to $24 \mathrm{mSv}$ have been reported for standard imaging protocols. The use of effective dose, however, is limited because it cannot be easily added to the absorbed dose to estimate total dose delivered to patient.

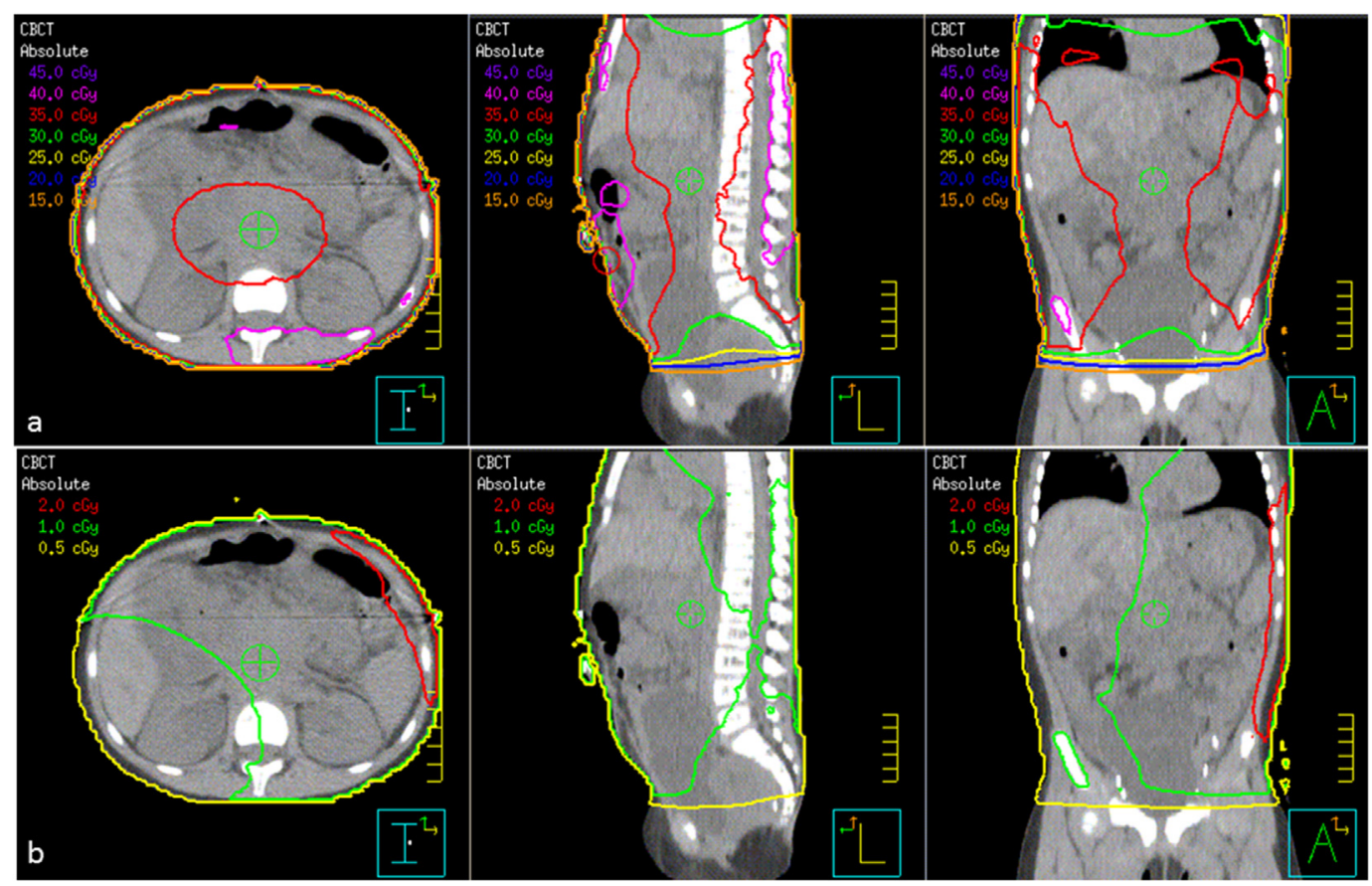

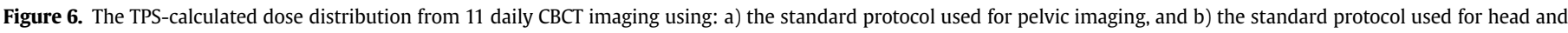
neck imaging demonstrating an 18 -fold reduction in dose. 
Measurements of CTDI for CBCT systems.

Computed Tomography Dose Index (CTDI) has been an established quantitative tool for dose measurements of CT scanners since its introduction in 1981 [93]. The CTDI measurements are made in polymethyl methacrylate (PMMA) cylinders of $15 \mathrm{~cm}$ in length and 16 and $32 \mathrm{~cm}$ in diameter (mimicking head and body) using $10 \mathrm{~cm}$ long ionization chambers. The usage of the CTDI, however, has become the subject of debate in recent years with the advent of multi-slice detectors with scan widths greater than $10 \mathrm{~cm}$ which result in primary radiation not registered completely by the chamber [9]. The International Electrotechnical Commission (IEC) [94], AAPM [95], and International Atomic Energy Agency (IAEA) [96] have proposed new and different approaches to measure CTDI which are beyond the scope of this review. The publications reviewed here use the traditional CTDI measurement methodology expanded to $\mathrm{CBCT}$, even though the same problem with limited chamber size exists as CBCT scan widths are often more than $10 \mathrm{~cm}$ $[18,21,25,33,46,82,97,98]$. In addition, CBCT scan widths may be longer than the $15 \mathrm{~cm}$ phantom length, hence some groups have stacked up two or three phantoms to cover the entire length of the CBCT scan [21,32]. There has also been a new terminology "Cone Beam Dose Index" or CBDI proposed for CTDI measurements on CBCT systems $[21,25,82]$.

The reported measured CTDI values for both Varian OBI and Elekta XVI systems are summarized in Table 6. The values tabulated here are normalized to the current-time product, i.e. divided by $100 \mathrm{~mA} \mathrm{~s}\left[\mathrm{CTDI}_{\mathrm{w}}\right.$ (mGy/100 mA s)]. Only the values obtained using the standard CTDI phantoms and $10 \mathrm{~cm}$ ion chamber are included in this table. A review of this data indicates obvious variations, which are due to differences in technique, cassette/field size, and bowtie filter with an average value of $4.6( \pm 2.6) \mathrm{mGy}$. The average value for head measurements is $5.8( \pm 3.1) \mathrm{mGy}$ and for body ones is $3.9( \pm 2.1)$ $m G y$. The values reported for Varian OBI are higher on average than those for Elekta XVI (5.6 vs. $3.3 \mathrm{mGy}$ ); although this cannot be used to draw conclusions on the relative dose output of the two systems as the data spans multiple versions of these systems.
A comparison of the reported cone beam $\mathrm{CTDI}_{\mathrm{w}}$ values in Table 6 with those reported for diagnostic fan beam CT scanners indicates generally lower values for CBCT scans than fan-beam CT ones. For example, Lee at al [99]. provided a database of normalized $\mathrm{CTDI}_{\mathrm{W}}$ values for all major manufacturers and reported $\mathrm{CTDI}_{\mathrm{W}}$ values between 7 and $24 \mathrm{mGy} / 100 \mathrm{~mA}$ s for the $120 \mathrm{kVp}$ beam, which is higher on average than that for CBCT as tabulated in Table 6. The reason for this difference is two-fold: The filtration used is CBCT systems is different than that in diagnostic CT units, and the photon fluence in CBCT is smaller than that in fan beam CT under similar $\mathrm{kVp}$ and $\mathrm{mAs}$ because the longitudinal profile in a CT beam does not drop-off as rapidly as that in CBCT.

\section{Conclusions}

Cone beam CT has become a standard imaging tool for patient localization in radiation therapy. Imaging dose from CBCT has traditionally been ignored as its magnitude has been deemed negligible comparing to therapeutic dose used to treat patients. However the daily use of this imaging modality prompted another look into the necessity and efficacy of measuring, documenting, and accounting for imaging dose. This clearly becomes an issue of concern if the magnitude of the imaging dose is significant compared to the therapeutic one. This review summarized the efforts made in measurement and calculation of the additional dose from this imaging modality and its potential effect on the overall dose delivered to patients during their course of radiation therapy treatment.

Since the onset of the use of CBCT units, there have been improvements by the manufacturers in reducing imaging dose, with the newer systems generally delivering lower dose than the older ones. Current CBCT systems use different methods, including collimator cassettes and field size-defining blades, to limit the extent of the x-ray beam. This has an effect on the total volume of the scan and consequently on the imaging dose to healthy tissues. The use of bowtie filters affects beam quality and often results in lower skin doses. Moreover, post-processing techniques are often

Table 6

Summary of $\mathrm{CTDI}_{\mathrm{w}}$ measurements for CBCT systems.

\begin{tabular}{|c|c|c|c|c|c|c|}
\hline & $\begin{array}{l}\text { Manufacturer } \\
\text { (Version if specified) }\end{array}$ & $\mathrm{kVp}$ & mAs/acquisition & $\begin{array}{l}\text { CTDI phantom } \\
\text { (Head or body) }\end{array}$ & $\begin{array}{l}\mathrm{CTDI}_{\mathrm{w}} \\
(\mathrm{mG} / 100 \mathrm{~mA} \mathrm{~s})\end{array}$ & Radiation field/filter \\
\hline \multirow[t]{2}{*}{ Sykes [18] } & Elekta XVI & 130 & 0.56 & Head & 8.5 & S20/no filter \\
\hline & (v3.1) & 100 & 0.25 & Head & 4.2 & S20/no filter \\
\hline Walter [46] & Elekta XVI & 120 & 1 & Body & 2.9 & M10 \\
\hline \multirow[t]{10}{*}{ Song [97] a } & Elekta XVI & 100 & 0.1 & Head & 2.8 & S20/no filter \\
\hline & & 120 & 1 & Body & 2.8 & L20/no filter \\
\hline & & 120 & 1 & Body & 3.7 & M20/no filter \\
\hline & & 120 & 1.6 & Body & 3.4 & M10/no filter \\
\hline & Varian OBI & 125 & 0.4 & Head & 6.7 & Full-fan/full-bowtie \\
\hline & & 125 & 0.4 & Body & 4.4 & Half-fan/half-bowtie \\
\hline & & 125 & 0.4 & Head & 11.5 & Full-fan/no filter \\
\hline & & 125 & 0.4 & Body & 7.5 & Half-fan/no filter \\
\hline & & 125 & 2 & Head & 6.6 & Full-bowtie/full-fan \\
\hline & & 125 & 2 & Body & 4.3 & Half-bowtie/half-fan \\
\hline \multirow[t]{4}{*}{ Osei [25] } & Varian OBI & 125 & 1 & Body & 3.2 & Full-fan/full-bowtie \\
\hline & & 125 & 1 & Body & 3.9 & Half-fan/half-bowtie \\
\hline & & 125 & 1 & Body & 8.3 & Full-fan/no filter \\
\hline & & 125 & 1 & Body & 7.5 & Half-fan/no filter \\
\hline \multirow[t]{6}{*}{ Hyer [82] } & Elekta XVI & 100 & 0.1 & Head & 2.7 & S20/no filter \\
\hline & $(\mathrm{v} 4.0)$ & 120 & 1.6 & Body & 1.6 & M20/bowtie \\
\hline & & 120 & 2.56 & Body & 1.5 & M10/bowtie \\
\hline & Varian OBI & 100 & 0.4 & Head & 3.6 & Full-fan/full-bowtie \\
\hline & (v1.4) & 110 & 0.4 & Body & 2.3 & Half-fan/half-bowtie \\
\hline & & 125 & 1.04 & Body & 3.2 & Half-fan/half-bowtie \\
\hline Falco [98] & $\begin{array}{l}\text { Elekta XVI } \\
\text { (v3.5) }\end{array}$ & 120 & 1.6 & Body & 2.4 & M10/bowtie \\
\hline
\end{tabular}

\footnotetext{
a 18 and $30 \mathrm{~cm}$ phantoms used in this study.
} 
used to obtain acceptable images with lower dose. The magnitude of the dose from the CBCT system is directly related to the currenttime product used in the image acquisition protocol. This value is usually pre-set within each clinical protocol but can be changed by the user. The size of the patient also affects the magnitude of the dose delivered at depth, especially for kilovoltage beams. A direct correlation has been observed between the patient size (indicated by BMI) and organ dose from kilovoltage imaging, especially since CBCT systems do not use automatic exposure control (AEC) adjustment, which is common in diagnostic imaging units. Equipping $\mathrm{CBCT}$ systems with AEC would help reducing imaging dose. In addition, there are methods that can be utilized by the user to reduce imaging dose including: (a) adjusting the start/stop angles of the x-ray source, potentially avoiding radio-sensitive organs, (b) reducing scan length by using smaller cassettes or field sizes, (c) using low-dose protocols to image, providing an acceptable image can be acquired.

If a high dose protocol is employed on a daily basis, it is possible to deliver, over the course of treatment, imaging dose to patient equal to one therapy dose fraction. In this case, it may be warranted to include the imaging dose in the planning process. It has been shown that this approach is feasible and that including imaging dose during plan optimization reduces the overall dose delivered to patient $[54,78,88]$. This, however, is not a trivial task and requires either use of Monte Carlo or commissioning of imaging beams in treatment planning systems. Currently, none of the commercially available systems are capable of computing dose from kilovoltage imaging beams. The capability of accounting for imaging dose added to therapeutic dose in IGRT should be implemented in commercial treatment planning systems to allow for full personalization of dose optimization.

As for the magnitude of the dose, as reported in this work the overall dose delivered from $\mathrm{kV} \mathrm{CBCT}$ ranges from a fraction of one to 4-5 cGy to internal organs, with values of up to $7 \mathrm{cGy}$ reported for skin dose. The dose varies depending on the protocol used, with protocols designed for head and neck imaging delivering doses at the low end of this range, and those designed for pelvic imaging delivering doses at the high end of it. The published data is often subject to large variations because of the differences in methods and phantoms used for imaging dose calculations. For example, AAPM TG-179 states that the imaging dose ranges from 0.2 to 2 cGy [100]. This range, however, is not entirely accurate as one can derive from Tables 1 and 2 of this review. MV CBCT, using the $6 \mathrm{MV}$ beam (TBL), often delivers higher doses than $\mathrm{kV}$ CBCT, with reported values of over $10 \mathrm{cGy}$ per fraction for high dose protocols, and accounting for this additional dose should be of high importance when a frequent CBCT scan approach is used. The MV CBCT dose can easily be calculated by the treatment planning systems and accounted for in patient treatment plans as shown in several publications $[54,55,75]$.

The peripheral dose from CBCT has been found to be at the same order of magnitude or smaller than that from leakage and scattered radiation from therapeutic beams. This could only be of concern when a radiosensitive electronic device is exposed to the imaging beam. Effective dose can be used to describe imaging dose with the limitation that it cannot be easily added to the absorbed dose, which is the common descriptor of dose in radiation therapy.

The CTDI values measured for CBCT systems can be used as an indicator of the average dose delivered by the unit by delivering the $\mathrm{x}$-rays to a cylindrical phantom in the central region of a scan. Since CTDI requires the use of a standard phantom for measurement, it can easily be compared between various manufacturers and the data tabulated in this review show the full extent of variations in dose due to different CBCT systems and settings. Due to the limitations of measuring CTDI values for $\mathrm{CBCT}$ systems, such as the limited length of the ion chamber and the phantom compared to the dimensions of the CBCT X-ray beam, it may be necessary to follow the recommendations of AAPM [95] and IEC [94], or more recently IAEA [96] on CT dosimetry of wide cone beam scanners and the adoption of the $\mathrm{CTDI}_{300}$ concept.

This review demonstrates that there is increasing awareness and strong interest in the evaluation of the concomitant dose from CBCT. With image guidance becoming a very important part of the radiotherapy treatment pathway of each patient, and with treatment adaptation based on a dose re-calculation on the actual patient treatment position becoming increasingly possible, it is very important that we implement the tools needed to control and manage the potentially detrimental effects of additional dose from on-board imaging devices. We hope that the data systematically collected in this work will provide useful reference material for clinicians and scientists to use in daily clinical practice and possibly accelerate the clinical implementation of solutions that are currently only available in the research environment.

\section{References}

[1] ACR. ACR-ASTRO. Practice guidelines for image-guided radiation therapy (IGRT). American College of Radiology, 2009; 2009.

[2] NHS. National Radiotherapy Implementation Group Report. Image Guided Radiotherapy (IGRT), Guidance for implementation and use, National Health Service. 2012.

[3] Jaffray DA, Langen KM, Mageras G, Dawson LA, Yan D, Edd RA, et al. Safety considerations for IGRT: executive summary. Pract Radiat Oncol 2013:3: $167-70$

[4] Zelefsky MJ, Kollmeier M, Cox B, Fidaleo A, Sperling D, Pei X, et al. Improved clinical outcomes with high-dose image guided radiotherapy compared with non-IGRT for the treatment of clinically localized prostate cancer. Int J Radiat Oncol Biol Phys 2012;84:125-9.

[5] Murphy MJ, Balter J, Balter S, BenComo JA, Das IJ, Jiang SB, et al. The management of imaging dose during image-guided radiotherapy: report of the AAPM Task Group 75. Med Phys 2007;34:4041.

[6] Kan MW, Leung LH, Wong W, Lam N. Radiation dose from cone beam computed tomography for image-guided radiation therapy. Int J Radiat Oncol Biol Phys 2008; 70:272-9.

[7] Lu B, Lu H, Palta J. A comprehensive study on decreasing the kilovoltage cone-beam CT dose by reducing the projection number. J Appl Clin Med Phys 2010;11:231-49.

[8] Sykes JR, Lindsay R, Iball G, Thwaites DI. Dosimetry of CBCT: methods, doses and clinical consequences. J Phys Conf Ser 2013:444.

[9] Kalender WA. Dose in X-ray computed tomography. Phys Med Biol 2014;59. R129-R50.

[10] Dougeni E, Faulkner K, Panayiotakis G. A review of patient dose and optimisation methods in adult and paediatric CT scanning. Eur J Radiology 2012;81:e665-83.

[11] Meeks SL, Harmon Jr JF, Langen KM, Willoughby TR, Wagner TH, Kupelian PA. Performance characterization of megavoltage computed tomography imaging on a helical tomotherapy unit. Med Phys 2005;32: 2673-81.

[12] Shah AP, Langen KM, Ruchala KJ, Cox A, Kupelian PA, Meeks SL. Patient dose from megavoltage computed tomography imaging. Int J Radiat Oncol Biol Phys 2008:70:1579-87.

[13] Fahrig R, Dixon R, Payne T, Morin RL, Ganguly A, Strobel N. Dose and image quality for a cone-beam C-arm CT system. Med Phys 2006;33:4541-50.

[14] Morant JJ, Salvad M, Herńandez-Giŕon I, Casanovas R, Ortega R, Calzado A. Dosimetry of a cone beam CT device for oral and maxillofacial radiology using Monte Carlo techniques and ICRP adult reference computational phantoms. Dentomaxillofacial Radiol 2013;42:92555893.

[15] Li G. Patient radiation dose and protection from cone-beam computed tomography. Imaging Sci Dent 2013;43:63-9.

[16] Matsuzaki Y, Fujii K, Kumagai M, Tsuruoka I, Mori S. Effective and organ doses using helical 4DCT for thoracic and abdominal therapies. J Radiat Res 2013;54:962-70.

[17] Ding GX, Malcolm AW. An optically stimulated luminescence dosimeter for measuring patient exposure from imaging guidance procedures. Phys Med Biol 2013;58:5885-97.

[18] Sykes JR, Amer A, Czajka J, Moore CJ. A feasibility study for image guided radiotherapy using low dose, high speed, cone beam X-ray volumetric imaging. Radiother Oncol 2005;77:45-52.

[19] Létourneau D, Wong JW, Oldham M, Gulam M, Watt L, Jaffray DA, et al. Conebeam-CT guided radiation therapy: Technical implementation. Radiother Oncol 2005;75:279-86. 
[20] Islam MK, Purdie TG, Norrlinger BD, Alasti H, Moseley DJ, Sharpe MB, et al Patient dose from kilovoltage cone beam computed tomography imaging in radiation therapy. Med Phys 2006;33:1573-82.

[21] Amer A, Marchant T, Sykes J, Czajka J, Moore C. Imaging doses from the elekta synergy X-ray cone beam CT system. Br J Radiology 2007;80:476-82.

[22] Wen N, Guan H, Hammoud R, Pradhan D, Nurushev T, Li S, et al. Dose delivered from Varian's CBCT to patients receiving IMRT for prostate cancer. Phys Med Biol 2007;52:2267-76.

[23] Gayou O, Parda DS, Johnson M, Miften M. Patient dose and image quality from mega-voltage cone beam computed tomography imaging. Med Phys 2007;34:499-506.

[24] Morin O, Gillis A, Descovich M, Chen J, Aubin M, Aubry JF, et al. Patient dose considerations for routine megavoltage cone-beam CT imaging. Med Phys 2007;34:1819-27.

[25] Osei EK, Schaly B, Fleck A, Charland P, Barnett R. Dose assessment from an online kilovoltage imaging system in radiation therapy. J Radiological Prot 2009;29:37-50.

[26] Winey B, Zygmanski P, Lyatskaya Y. Evaluation of radiation dose delivered by cone beam CT and tomosynthesis employed for setup of external breast irradiation. Med Phys 2009;36:164-73.

[27] Marinello G, Mege JP, Besse MC, Kerneur G, Lagrange JL. [Prostate radiation therapy: in vivo measurement of the dose delivered by kV-CBCT]. Cancer radiotherapie. J De La Soc Francaise De Radiotherapie Oncol 2009;13:353-7.

[28] Isambert A, Ferreira IH, Bossi A, Beaudré A, Nicula LE, Lefkopoulos D. Dose delivered to the patient by megavoltage cone beam computed tomography imaging. Dose Délivrée Patient lors l'acquisition d'images Tomogr Conique Haute Énergie 2009; 13:358-64.

[29] Tomic N, Devic S, Deblois F, Seuntjens J. Reference radiochromic film dosimetry in kilovoltage photon beams during CBCT image acquisition. Med Phys 2010;37:1083-92.

[30] Tomic N, Devic S, Deblois F, Seuntjens J. Comment on reference radiochromic film dosimetry in kilovoltage photon beams during CBCT image acquisition. Med Phys 2010;37:3008.

[31] Hyer DE, Serago CF, Kim S, Li JG, Hintenlang DE. An organ and effective dose study of XVI and OBI cone-beam CT systems. J Appl Clin Med Phys 2010;11: $181-97$.

[32] Palm Å Nilsson E, Herrnsdorf L. Absorbed dose and dose rate using the varian OBI 1.3 and 1.4 CBCT system. J Appl Clin Med Phys 2010;11:229-40.

[33] Cheng HCY, Wu VWC, Liu ESF, Kwong DLW. Evaluation of radiation dose and image quality for the varian cone beam computed tomography system. Int J Radiat Oncol Biol Phys 2011;80:291-300.

[34] Dufek V, Horakova I, Novak L. Organ and effective doses from verification techniques in image-guided radiotherapy. Radiat Prot Dosim 2011;147: 277-80.

[35] Abou-Elenein HS, Attalla EM, Ammar H, Eldesoky I, Farouk M, Zaghloul MS Megavoltage cone beam computed tomography: commissioning and evaluation of patient dose. J Med Phys 2011;36:205-12.

[36] Quinn A, Holloway L, Cutajar D, Hardcastle N, Rosenfeld A, Metcalfe P. Megavoltage cone beam CT near surface dose measurements: potential implications for breast radiotherapy. Med Phys 2011;38:6222-7.

[37] Shah A, Aird E, Shekhdar J. Contribution to normal tissue dose from concomitant radiation for two common $\mathrm{kV}$-CBCT systems and one MVCT system used in radiotherapy. Radiother Oncol 2012;105:139-44.

[38] Stock M, Palm A, Altendorfer A, Steiner E, Georg D. IGRT induced dose burden for a variety of imaging protocols at two different anatomical sites. Radiother Oncol 2012;102:355-63.

[39] Hälg RA, Besserer J, Schneider U. Systematic measurements of whole-body imaging dose distributions in image-guided radiation therapy. Med Phys 2012;39:7650-61.

[40] Kim DW, Chung WK, Yoon M. Imaging doses and secondary cancer risk from kilovoltage cone-beam ct in radiation therapy. Health Phys 2013;104 499-503.

[41] Giaddui T, Cui Y, Galvin J, Yu Y, Xiao Y. Comparative dose evaluations between XVI and OBI cone beam CT systems using gafchromic XRQA2 film and nanoDot optical stimulated luminescence dosimeters. Med Phys 2013;40: 062102.

[42] Alvarado R, Booth JT, Bromley RM, Gustafsson HB. An investigation of image guidance dose for breast radiotherapy. J Appl Clin Med Phys 2013;14:25-38.

[43] Moon YM, Kim HJ, Kwak DW, Kang YR, Lee MW, Ro TI, et al. Effective dose measurement for cone beam computed tomography using glass dosimeter Nucl Eng Technol 2014;46:255-62.

[44] Nobah A, Aldelaijan S, Devic S, Tomic N, Seuntjens J, Al-Shabanah M, et al Radiochromic film based dosimetry of image-guidance procedures on different radiotherapy modalities. J Appl Clin Med Phys 2014;15:229-39.

[45] Flynn RT, Hartmann J, Bani-Hashemi A, Nixon E, Alfredo R, Siochi C, et al. Dosimetric characterization and application of an imaging beam line with a carbon electron target for megavoltage cone beam computed tomography. Med Phys 2009;36:2181-92.

[46] Walter C, Boda-Heggemann J, Wertz H, Loeb I, Rahn A, Lohr F, et al. Phanton and in-vivo measurements of dose exposure by image-guided radiotherapy (IGRT): MV portal images vs. kV portal images vs. cone-beam CT. Radiother Oncol 2007;85:418-23.

[47] Jeng SC, Tsai CL, Chan WT, Tung CJ, Wu JK, Cheng JCH. Mathematical estimation and in vivo dose measurement for cone-beam computed tomography on prostate cancer patients. Radiother Oncol 2009;92:57-61.
[48] Ding GX, Coffey CW. Beam characteristics and radiation output of a kilovoltage cone-beam CT. Phys Med Biol 2010;55:5231-48.

[49] Ding GX, Duggan DM, Coffey CW. Accurate patient dosimetry of kilovoltage cone-beam CT in radiation therapy. Med Phys 2008;35:1135-44.

[50] Spezi E, Downes P, Radu E, Jarvis R. Monte Carlo simulation of an X-ray volume imaging cone beam CT unit. Med Phys 2009:36:127-36.

[51] Downes P, Jarvis R, Radu E, Kawrakow I, Spezi E. Monte Carlo simulation and patient dosimetry for a kilovoltage cone-beam CT unit. Med Phys 2009;36: 4156-67.

[52] Alaei P, Ding G, Guan H. Inclusion of the dose from kilovoltage cone beam CT in the radiation therapy treatment plans. Med Phys 2010;37:244-8.

[53] Alaei P, Spezi E. Commissioning kilovoltage cone-beam CT beams in a radiation therapy treatment planning system. J Appl Clin Med Phys 2012;13: 19-33.

[54] Miften M, Gayou O, Reitz B, Fuhrer R, Leicher B, Parda DS. IMRT planning and delivery incorporating daily dose from mega-voltage cone-beam computed tomography imaging. Med Phys 2007;34:3760-7.

[55] Morin O, Chen J, Aubin M, Gillis A, Aubry JF, Bose S, et al. Dose calculation using megavoltage cone-beam CT. Int J Radiat Oncol Biol Phys 2007;67: $1201-10$.

[56] Faddegon BA, Wu V, Pouliot J, Gangadharan B, Bani-Hashemi A. Low dose megavoltage cone beam computed tomography with an unflattened 4 MV beam from a carbon target. Med Phys 2008;35:5777-86.

[57] Dzierma Y, Nuesken F, Otto W, Alaei P, Licht N, Rübe C. Dosimetry of an inline kilovoltage imaging system and implementation in treatment planning. Int J Radiat Oncol Biol Phys 2014;88:913-9.

[58] Chow JCL, Leung MKK, Islam MK, Norrlinger BD, Jaffray DA. Evaluation of the effect of patient dose from cone beam computed tomography on prostate IMRT using Monte Carlo simulation. Med Phys 2008;35:52-60.

[59] Deng J, Chen Z, Roberts KB, Nath R. Kilovoltage imaging doses in the radiotherapy of pediatric cancer patients. Int J Radiat Oncol Biol Phys 2012;82: 1680-8.

[60] Deng J, Chen Z, Yu JB, Roberts KB, Peschel RE, Nath R. Testicular doses in image-guided radiotherapy of prostate cancer. Int J Radiat Oncol Biol Phys 2012;82:e39-47.

[61] Gu J, Bednarz B, Xu GX, Jiang SB. Assessment of patient organ doses and effective doses using the VIP-Man adult male phantom for selected conebeam CT imaging procedures during image guided radiation therapy. Radiat Prot Dosim 2008;131:431-43.

[62] Walters BRB, Ding GX, Kramer R, Kawrakow I. Skeletal dosimetry in cone beam computed tomography. Med Phys 2009;36:2915-22.

[63] Ding GX, Coffey CW. Radiation dose from kilovoltage cone beam computed tomography in an image-guided radiotherapy procedure. Int J Radiat Oncol Biol Phys 2009;73:610-7.

[64] Ding A, Gu J, Trofimov AV, Xu XG. Monte Carlo calculation of imaging doses from diagnostic multidetector CT and kilovoltage cone-beam CT as part of prostate cancer treatment plans. Med Phys 2010;37:6199-204.

[65] Oiu Y, Popescu IA, Duzenli C. Moiseenko V. Mega-voltage versus kilo-voltage cone beam CT used in image guided radiation therapy: comparative study of microdosimetric properties. Radiat Prot Dosim 2011;143:477-80.

[66] Spezi E, Downes P, Jarvis R, Radu E, Staffurth J. Patient-specific threedimensional concomitant dose from cone beam computed tomography exposure in image-guided radiotherapy. Int J Radiat Oncol Biol Phys 2012;83:419-26.

[67] Oiu Y, Moiseenko V, Aquino-Parsons C, Duzenli C. Equivalent doses for gynecological patients undergoing IMRT or RapidArc with kilovoltage cone beam CT. Radiother Oncol 2012;104:257-62.

[68] Zhang Y, Yan Y, Nath R, Bao S, Deng J. Personalized assessment of kV cone beam computed tomography doses in image-guided radiotherapy of pediatric cancer patients. Int J Radiat Oncol Biol Phys 2012;83: $1649-54$.

[69] Ding GX, Munro P. Radiation exposure to patients from image guidance procedures and techniques to reduce the imaging dose. Radiother Oncol 2013;108:91-8.

[70] Son K, Cho S, Kim JS, Han Y, Ju SG, Choi DH. Evaluation of radiation dose to organs during kilovoltage cone-beam computed tomography using Monte Carlo simulation. J Appl Clin Med Phys 2014;15:295-302.

[71] Spezi E, Volken W, Frei D, Fix MK. A virtual source model for Kilo-voltage cone beam CT: source characteristics and model validation. Med Phys 2011;38:5254-63.

[72] Montanari D, Scolari E, Silvestri C, Graves YJ, Yan H, Cervino L, et al. Comprehensive evaluations of cone-beam CT dose in image-guided radiation therapy via GPU-based Monte Carlo simulations. Phys Med Biol 2014;59: 1239-53.

[73] Peng LC, Yang CCJ, Sim S, Weiss M, Bielajew A. Dose comparison of megavoltage cone-beam and orthogonal-pair portal images. J Appl Clin Med Phys 2007;8:10-20.

[74] VanAntwerp AE, Raymond SM, Addington MC, Gajdos S, Vassil A, Xia P. Dosimetric evaluation between megavoltage cone-beam computed tomography and body mass Index for Intracranial, thoracic, and pelvic localization. Med Dosim 2011;36:284-91.

[75] Akino Y, Koizumi M, Sumida I, Takahashi Y, Ogata T, Ota S, et al. Megavoltage cone beam computed tomography dose and the necessity of reoptimization for imaging dose-integrated intensity-modulated radiotherapy for prostate cancer. Int J Radiat Oncol Biol Phys 2012;82:1715-22. 
[76] Zabel-du Bois A, Nill S, Ulrich S, Oelfke U, Rhein B, Haering P, et al. Dosimetric integration of daily mega-voltage cone-beam CT for image-guided intensitymodulated radiotherapy. Strahlenther Onkol 2012;188:120-6.

[77] Beltran C, Lukose R, Gangadharan B, Bani-Hashemi A, Faddegon BA. Image quality \& dosimetric property of an investigational imaging beam line MVCBCT. J Appl Clin Med Phys 2009;10:37-48.

[78] Alaei P, Spezi E, Reynolds M. Dose calculation and treatment plan optimization including imaging dose from kilovoltage cone beam computed tomography. Acta Oncol 2014;53:839-44.

[79] Ding GX, Pawlowski JM, Coffey CW. A correction-based dose calculation algorithm for kilovoltage x rays. Med Phys 2008;35:5312-6.

[80] Pawlowski JM, Ding GX. An algorithm for kilovoltage x-ray dose calculations with applications in kV-CBCT scans and 2D planar projected radiographs. Phys Med Biol 2014;59:2041-58.

[81] Pawlowski JM, Ding GX. A new approach to account for the mediumdependent effect in model-based dose calculations for kilovoltage x-rays. Phys Med Biol 2011;56:3919-34.

[82] Hyer DE, Hintenlang DE. Estimation of organ doses from kilovoltage conebeam CT imaging used during radiotherapy patient position verification. Med Phys 2010:37:4620-6.

[83] Nelson AP, Ding GX. An alternative approach to account for patient organ doses from imaging guidance procedures. Radiother Oncol 2014;112:112-8.

[84] Perks JR, Lehmann J, Chen AM, Yang CC, Stern RL, Purdy JA Comparison of peripheral dose from image-guided radiation therapy (IGRT) using $\mathrm{kV}$ cone beam CT to intensity-modulated radiation therapy (IMRT). Radiother Oncol 2008;89:304-10.

[85] Jia MX, Zhang X, Li N, Wang EY, Liu DW, Cai WS. Peripheral dose from megavoltage cone-beam CT imaging for nasopharyngeal carcinoma imageguided radiation therapy. J Appl Clin Med Phys 2012;13:3-11.

[86] Ding GX, Munro P, Pawlowski J, Malcolm A, Coffey CW. Reducing radiation exposure to patients from kV-CBCT imaging. Radiother Oncol 2010;97: 585-92.

[87] Roxby P, Kron T, Foroudi F, Haworth A, Fox C, Mullen A, et al. Simple methods to reduce patient dose in a Varian cone beam CT system for delivery verification in pelvic radiotherapy. Br J Radiology 2009;82:855-9.
[88] Grelewicz Z, Wiersma RD. Combined MV + kV inverse treatment planning for optimal kV dose incorporation in IGRT. Phys Med Biol 2014:59:1607-21.

[89] Alaei P, Spezi E, Ehler E, Dusenbery K. SU-E-J-08: assessing and minimizing the dose from KV cone beam CT to pediatric patients undergoing radiation therapy. Med Phys 2013;40:151.

[90] Potts R, Oatey M. An investigation of the concomitant doses from cone beam CT and CT simulation in radiotherapy. IFMBE Proc 2009;25:41-4.

[91] ICRP. 1990 Recommendations of the international commission on radiological Protection. 1990. ICRP publication 60.

[92] ICRP. The 2007 Recommendations of the international commission on radiological Protection. 2007. ICRP publication 103, 2007.

[93] Shope TB, Gagne RM, Johnson GC. A method for describing the doses delivered by transmission X-ray computed tomography. Med Phys 1981;8: 488-95.

[94] IEC. Medical electrical equipment - Part 2-44: Particular requirements for the basic safety and essential performance of X-ray equipment for computed tomography. 2009. International Electrotechnical Commission.

[95] AAPM. Comprehensive methodology for the evaluation of radiation dose in X-ray computed tomography, report of task group 111. Am Assoc Phys Med 2010. ISBN: 978-1-888340-94-5, ISSN: 0271-7344.

[96] IAEA. Status of computed tomography dosimetry for wide cone beam scanners. International Atomic Energy Agency; 2011.

[97] Song WY, Kamath S, Ozawa S, Al Ani S, Chvetsov A, Bhandare N, et al. A dose comparison study between $\mathrm{XVI}^{\circledR}$ and $\mathrm{OBI}{ }^{\circledR}$ CBCT systems. Med Phys 2008;35: 480-6.

[98] Falco MD, Fontanarosa D, Miceli R, Carosi A, Santoni R, D'Andrea M. Preliminary studies for a CBCT imaging protocol for Offline organ motion analysis: registration software validation and CTDI measurements. Med Dosim 2011;36:91-101.

[99] Lee E, Lamart S, Little MP, Lee C. Database of normalised computed tomography dose index for retrospective CT dosimetry. J Radiological Prot 2014;34 363-88.

[100] Bissonnette JP, Balter PA, Dong L, Langen KM, Lovelock DM, Miften M. Quality assurance for image-guided radiation therapy utilizing CT-based technologies: a report of the AAPM TG-179. Med Phys. 2012;39:1946-63. 